\title{
Ultrafast femtosecond-laser-induced fiber Bragg gratings in air-hole microstructured fibers for high-temperature pressure sensing
}

\author{
Charles M. Jewart, ${ }^{1}$ Qingqing Wang, ${ }^{1}$ John Canning, ${ }^{2}$ Dan Grobnic,,${ }^{3}$ Stephen J. Mihailov, ${ }^{3}$ and \\ Kevin P. Chen ${ }^{1, *}$ \\ ${ }^{1}$ Department of Electrical Engineering, University of Pittsburgh, Pittsburgh, Pennsylvania 15261, USA \\ ${ }^{2}$ Interdisciplinary Photonics Laboratories, School of Chemistry, University of Sydney, Sydney, Australia, NSW 1430 \\ ${ }^{3}$ Communication Research Center Canada, 3701 Carling Avenue, Ottawa, Ontario K2H 8S2, Canada \\ *Corresponding author: kchen@engr.pitt.edu
}

Received January 13, 2010; accepted February 13, 2010; posted March 23, 2010 (Doc. ID 122604); published April 29, 2010

We present fiber Bragg grating pressure sensors in air-hole microstructured fibers for high-temperature operation above $800^{\circ} \mathrm{C}$. An ultrafast laser was used to inscribe Type II grating in two-hole optical fibers. The fiber Bragg grating resonance wavelength shift and peak splits were studied as a function of external hydrostatic pressure from 15 psi to 2000 psi. The grating pressure sensor shows stable and reproducible operation above $800^{\circ} \mathrm{C}$. We demonstrate a multiplexible pressure sensor technology for a high-temperature environment using a single fiber and a single-fiber feedthrough. (c) 2010 Optical Society of America

OCIS codes: $060.2370,060.4005,120.5475,120.6780$.

Pressure sensors operated at high temperature above $500^{\circ} \mathrm{C}$ have many important applications in the energy industry. They ensure safe and efficient energy production during operations of gas turbines, coal boilers, nuclear power plants, and others. The hightemperature environment presents unique challenges to sensing systems. It not only requires robust sensor elements but also demands reliable packaging and wiring techniques rated for high-temperature environments.

Fiber-based optical sensors have been considered good candidates for applications within harsh environments. For example, high-temperature pressure sensors based on a Fabry-Perot interferometer (FPI) have been successfully demonstrated [1,2], and fiber pressure sensors with operating temperatures up to $800^{\circ} \mathrm{C}$ have been achieved using sapphire fibers [1]. On the other hand, conventional fiber Bragg grating (FBG) sensors are generally considered unsuitable for high-temperature operation. Although FBG pressure sensors have been fabricated using UV laser writing, their operational temperature was limited to below $300^{\circ} \mathrm{C}$ owing to the poor thermal stability of the UV-induced refractive index change [3,4].

In contrast to interferometer-based fiber sensors, FBG-based sensors are readily multiplexible, and the fabrication and the packaging of FBG sensors are relatively simple. They can be produced reliably and in large quantities using a phase mask writing technique. These highly desirable advantages have resulted in significant efforts to improve the hightemperature stability of FBG sensors. In particular, the adaptation of novel fibers, such as nitrogen-doped fiber [5], or novel fabrication techniques, such as ultrafast laser writings [6], has potentially improved the operational temperature of fiber grating devices to be on par with FPI-based sensors, approaching or exceeding $800^{\circ} \mathrm{C}$.

In this Letter, we apply femtosecond-pulsed ultrafast laser writing to produce high-temperature stable FBG in two-hole fibers for pressure sensing. This work demonstrates a significant improvement of the operational temperature of FBG pressure sensors in air-hole microstructured fiber to over $800^{\circ} \mathrm{C}$. A large number of high-temperature FBG pressure sensors in microstructured fibers can be fabricated in one fiber using a simple phase mask approach. The multiplexed fiber sensor array in a single fiber can be serviced by a single reading system employing a single fiber feedthrough, which will dramatically reduce the wiring complexity of a sensor network for high-temperature applications.

Air-hole fibers used in this work are $220 \mu \mathrm{m}$ diameter two-hole fibers with two $90 \mu \mathrm{m}$ air holes as shown in the inset of Fig. 1. When a hydrostatic pressure is applied to a twin-hole fiber, both air holes deform under the external pressure. The deformation of the air holes transfers the external pressure into internal stress in the fiber core. The pressure induces birefringence in the fiber, which can be measured by an FBG inscribed into the fiber core. To maximize the sensitivity of FBG sensors, two-hole fibers with a fiber core just $1 \mu \mathrm{m}$ from the edge of one air hole was

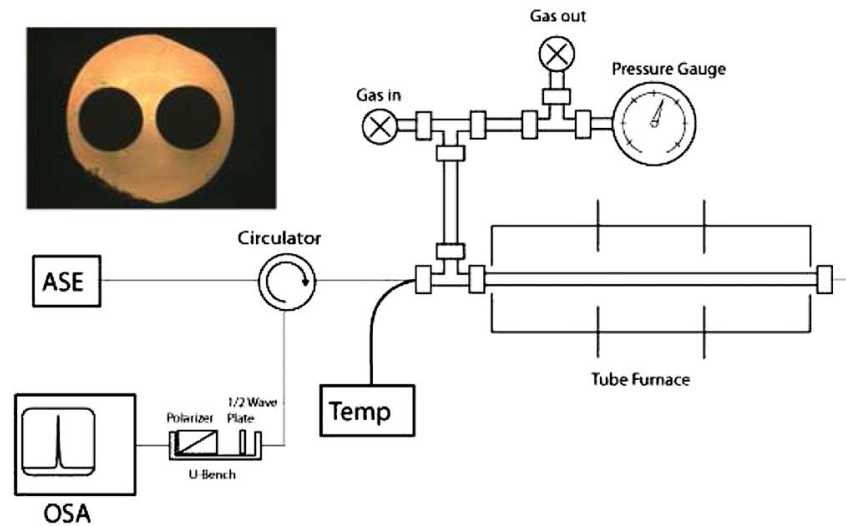

Fig. 1. (Color online) Schematic of high-temperature pressure-testing setup, the optical microscope cross-section photo is shown as an inset. 
chosen for the FBG inscription using an ultrafast laser.

The hydrostatic pressure testing was performed in a sealed stainless steel tube, as shown in Fig. 1. High-pressure nitrogen gas was introduced in the testing tube using a T-fitting. A two-hole fiber containing an FBG was fed through the tube and sealed at both end of tube using ceramic ferrules (Restek). The section of tube containing the FBG was heated in a tube furnace. To monitor the FBG response, the two-hole fiber was fusion spliced to the output port of a fiber circulator. A randomly polarized broadband amplified spontaneous emission source was connected to the input port of the circulator, and reflection spectra of the FBG were monitored by an optical spectrum analyzer (OSA, Ando 6317). To monitor the birefringence change, a U-bench was inserted in front of the OSA. A half-wave plate and a GlenThomson polarizer were placed in the U-bench. By rotating the wave plate, the polarizer can select one of two orthogonal polarized modes of the fiber.

FBGs used in this experiment were written by a kilohertz femtosecond Ti:sapphire laser at $800 \mathrm{~nm}$ using direct writing through a phase mask. The detailed writing procedure can be found in [6]. The hydrostatic pressure testing was performed using nitrogen gas. FBG responses were monitored as a function of external pressure from 15 to 2000 psi. Figure 2 shows the FBG spectra evolution from 23 to $858^{\circ} \mathrm{C}$ and cooled back to $23^{\circ} \mathrm{C}$ at room pressure of 15 psi. The FBG peak shifts linearly as a function of time at a slope of $14.9 \mathrm{pm} /{ }^{\circ} \mathrm{C}$ for both heating and cooling cycles without hysteresis. The unpolarized reflection spectrum shows a wide $1.2 \mathrm{~nm} 3 \mathrm{~dB}$ FWHM with a double-shoulder feature.

The heating and cooling cycles were repeated three times over a period of 3 days. The FBG withstood the high-temperature test; both shape and strength of the FBG spectra were not weakened or altered by the heating, as shown in the inset of Fig. 2. These results demonstrate the high-temperature robustness of FBGs in microstructured fiber fabricated by the ul-

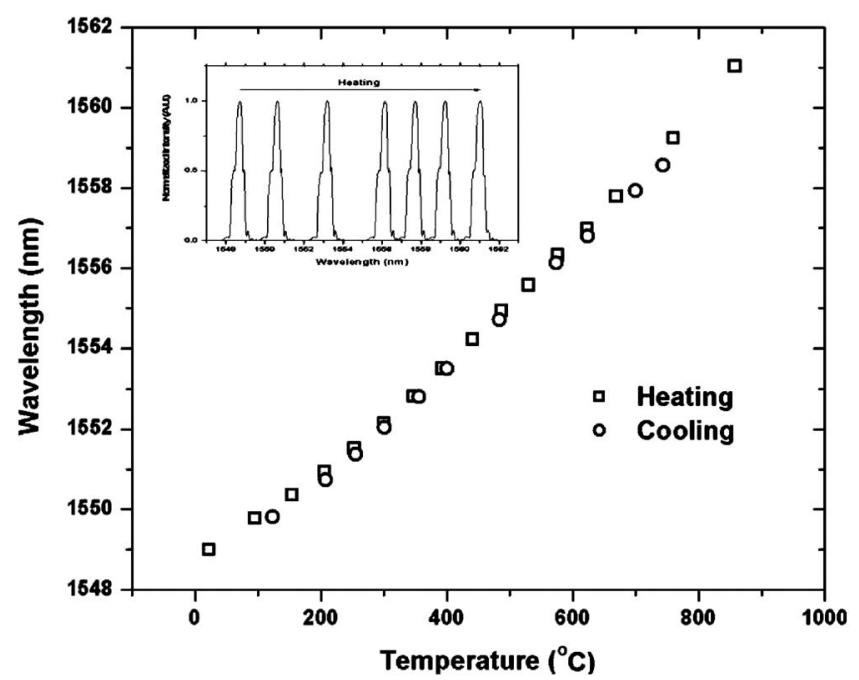

Fig. 2. FBG response as a function of temperature at room pressure. The FBG reflection spectra peak at 23, 198, 383, $570,666,760$, and $858^{\circ} \mathrm{C}$ are shown as an inset. trafast laser irradiation. These results are also consistent with FBGs inscribed by the same laser in standard optical fibers (SMF-28) [6].

The result of pressure testing at $858^{\circ} \mathrm{C}$ is shown in Fig. 3, in which the unpolarized FBG spectrum is plotted together with the FBG spectra of the two orthogonal polarization eigenstates at both $15 \mathrm{psi}$ [Fig. 3(a)] and 2000 psi [Fig. 3(b)]. The ultrafast laser writing process induces a larger birefringence of 1.9 $\times 10^{-4}$ in fiber [7]. This is in contrast with the $<10^{-5}$ intrinsic birefringence of the fiber. The large laserinduced birefringence separated the FBG peaks for both orthogonal polarization eigenstates, which is the origin of the double-shoulder feature of the unpolarized FBG spectra. To confirm this, reflection spectra at two orthogonal polarizations were combined and normalized to compute the unpolarized FBG spectrum. The shape of the calculated spectrum matches well with the measured unpolarized spectrum, as shown in Fig. 3.

There is a slight lateral shift between the calculated unpolarized reflection spectrum and the measured one. This is probably because the unpolarized spectrum and two polarized spectra were measured separately at different times. The temperature drift

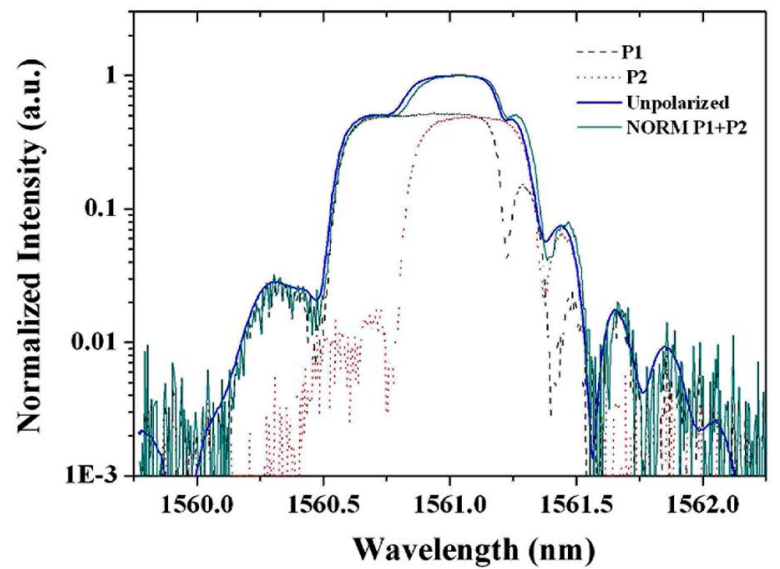

(a)

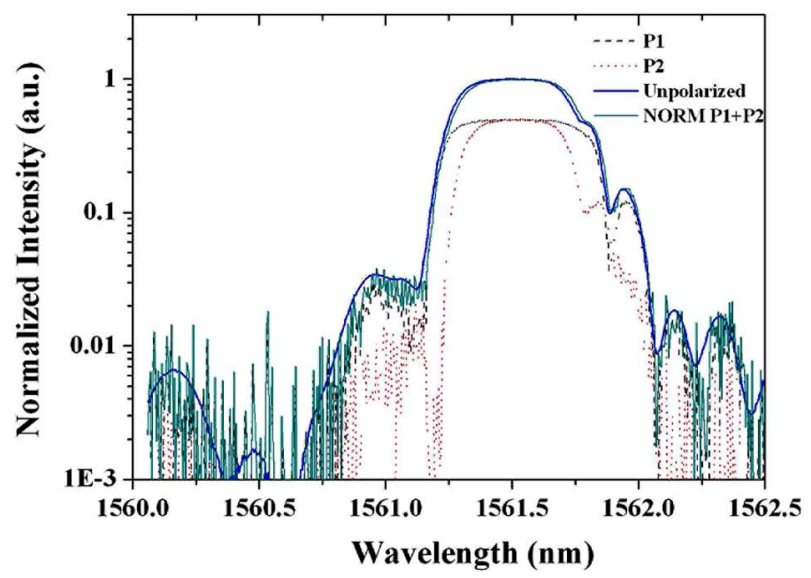

(b)

Fig. 3. (Color online) Normalized FBG reflection spectra at $858^{\circ} \mathrm{C}$ for two orthogonal polarizations (P1 and P2). The unpolarized reflection spectrum is also plotted together with the normalized combined $\mathrm{P} 1+\mathrm{P} 2$ spectrum for (a) 15 psi and (b) 2000 psi. 
between two measurements was a likely cause for this slight mismatch. At $858^{\circ} \mathrm{C}$, the fiber birefringence induced a $0.19 \mathrm{~nm}$ peak split between the two orthogonal polarizations. The increase of hydrostatic pressure to 2000 psi shifts both polarization peaks toward the longer wavelength. It also alters the effective index of one polarization more than that of the other one, which leads to a birefringence reduction and the FBG peak split from 0.19 to $0.006 \mathrm{~nm}$. The $0.006 \mathrm{~nm}$ split was obtained from a Gaussian fitting. The response of the FBG peak separation to the external fiber pressure can be used to measure pressure at high temperature. Figure 4 shows the correlation between FBG peak separation from two orthogonal modes and the external pressure at 858 and $199^{\circ} \mathrm{C}$. The FBG peak splits at both temperatures exhibits a linear trend as a function of external pressure. The linear trend levels off between 1500 and 2000 psi. This could be due to the measurement inaccuracy to determine small birefringence using FBGs with wide bandwidth, as shown in Fig. 3. Indeed, the deviation from the linear trend was not observed in an FBG in the same kind of two-hole fibers with a narrow linewidth [8].

Figure 5 shows a FBG inscribed in the same kind of two-hole fiber using a $\mathrm{KrF} 248 \mathrm{~nm}$ excimer laser. Using this mature laser technique, an FBG with $\sim 0.1 \mathrm{~nm}$ FWHM can be inscribed in two-hole fiber without increasing the intrinsic fiber birefringence $\left(<10^{-5}\right)$. At room temperature, the application of 1800 psi external pressure moves the FBG peaks from both polarization eigenstates toward longer wavelengths. At the same time, the pressure-induced birefringence produces a clear separation of the two FBG peaks at $0.16 \mathrm{~nm}$ under the 1800 psi. Further pressure experiment reveals that resonant wavelengths of UV-induced FBG for both polarizations show a linear dependence to the external pressure over the entire range of testing pressure from 15 to 1900 psi [8].

The maximum pressure used to test the FBG sensor was limited by our $\mathrm{N}_{2}$ tank pressure. It has been shown that air-hole silica fibers are able to sustain pressures up to 10000 psi [9]. In combination with the high-temperature robustness demonstrated in

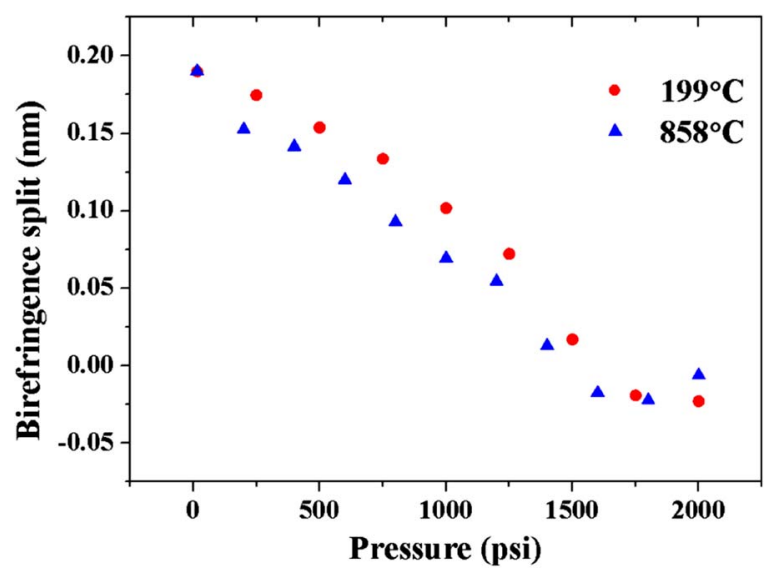

Fig. 4. (Color online) FBG birefringence vs. pressure at $199^{\circ} \mathrm{C}$ and $858^{\circ} \mathrm{C}$ respectively.

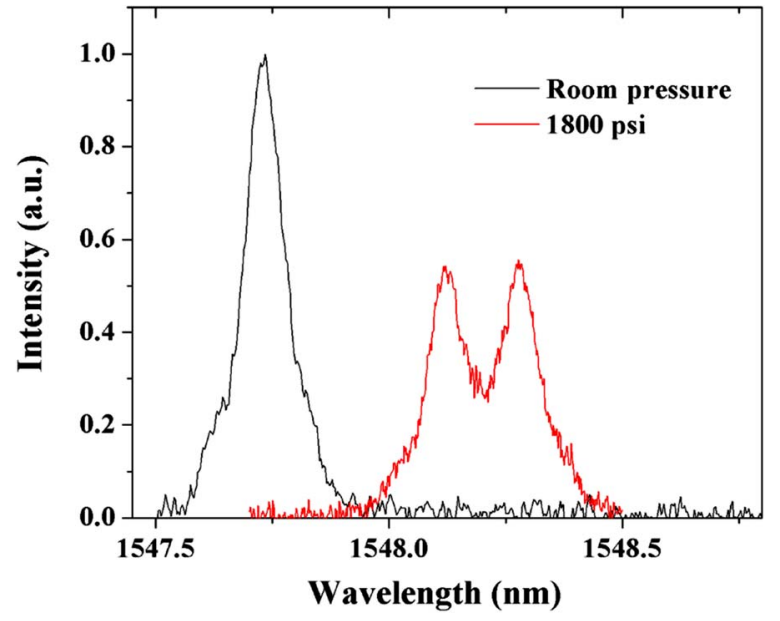

Fig. 5. (Color online) FBG inscribed by a $\mathrm{KrF} 248-\mathrm{nm}$ excimer laser in the same type of two-hole fiber and its response to external pressure at the room temperature.

this Letter, FBG written in microstructured fibers by an ultrafast laser can be an appealing hightemperature pressure-sensing technology for fossil fuel and nuclear energy applications. To enhance the sensitivity, future improvement should be made by reducing the linewidth of FBGs written by ultrafast lasers.

In summary, this Letter applies the ultrafast laser fabrication technique to produce high-temperature stable FBG pressure sensors in air-structured optical fibers for energy industry applications. In comparison with other fiber optical pressure sensors, FBGs inscribed by the ultrafast laser in air-hole fibers can be produced in large quantities using a phase mask writing technique. Hundreds of such FBG pressure sensors can be multiplexed in a single fiber and serviced by one fiber sensor reading unit using a singlefiber feedthrough.

This work was supported by the National Science Foundation (NSF) (CMMI-0644681 and CMMI0900564). C. M. Jewart and Q. Q. Wang made equal contributions to this work.

\section{References}

1. H. Xiao, J. Deng, Z. Wang, W. Huo, P. Zhang, M. Luo, G. R. Pickrell, R. G. May, and A. Wang, Opt. Eng. 44, 054403 (2005).

2. Y. Zhu, K. L. Cooper, G. R. Pickrell, and A. Wang, J. Lightwave Technol. 24, 861 (2006).

3. S. Kreger, S. Calvert, and E. Udd, in Optical Fiber Sensors Conference (Optical Society of America, 2002), pp. 355-358.

4. T. Yamate, R. T. Ramos, R. J. Schroeder, and E. Udd, Proc. SPIE 4185, 628 (2000).

5. O. V. Butov, E. M. Dianov, and K. M. Golant, Meas. Sci. Technol. 17, 975 (2006).

6. D. Grobnic, C. W. Smelser, S. J. Milailov, and R. B. Walker, Meas. Sci. Technol. 17, 1009 (2006).

7. P. Lu, D. Grobnic, and S. J. Mihailov, J. Lightwave Technol. 25, 779 (2007).

8. C. Jewart, D. Xu, K. P. Chen, and J. Canning, Proc. SPIE 7004, 70041Z (2008).

9. R. Clowes, S. Syngellakis, and M. N. Zervas, IEEE Photon. Technol. Lett. 10, 857 (1998). 